\title{
Publication Productivity in Nuclear Medicine
}

\author{
Cheryl McKellar ${ }^{1}$ and Geoff Currie ${ }^{1,2}$ \\ ${ }^{I}$ Faculty of Science, Charles Sturt University, Wagga Wagga, New South Wales, Australia; and ${ }^{2}$ Faculty of Medicine and Health \\ Sciences, Macquarie University, Sydney, New South Wales, Australia
}

Publications form the knowledge base of any profession. Patterns in professional publications provide insight into the profession's maturity and global status. To our knowledge, publication productivity in nuclear medicine technology has not been reported. A recent study on publication productivity in radiography and radiation therapy provided interesting insight; however, a sampling bias resulted in study flaws. Methods: The most productive medical radiation technologists were determined by collecting data from 7 key, international peer-reviewed journals for the medical radiation sciences over a 5-y period. A full list of the technologists' publications, for the 5-y period, was obtained using a PubMed and ResearchGate search, and the authors were analyzed. Results: In total, 165 medical radiation technologists were identified who had published 3 or more articles between 2009 and 2013. Of these authors, 55.2\% (91/165) were radiographers, $35.2 \%(58 / 165)$ were radiation therapists, and $9.6 \%(16 / 165)$ were nuclear medicine technologists. Overall, the majority of the most prolific authors were academics (104/165; $63.0 \%$ ). After we applied a correction factor (the productivity per member of the registered workforce), radiography had the fewest authors publishing, compared with the relative workflow sizes. Conclusion: Nuclear medicine technologists demonstrated a high degree of productivity both absolutely and relatively. Consequently, nuclear medicine technologists have a productive research culture and command a large footprint within and outside the key medical radiation science journals.

Key Words: publication; productivity; patterns, nuclear medicine; medical radiation science

J Nucl Med Technol 2015; 43:122-128

DOI: $10.2967 /$ jnmt.115.156307

$\mathbf{N}$ uclear medicine technology is a relatively young profession with an expanding knowledge economy. Indeed, the profession and professionalism have only recently been defined, and this development has rapidly evolved to the current position of advanced practice. Nonetheless, the professional status of nuclear medicine technology is not universal, with developing and undeveloped economies not demonstrating the same scope

\footnotetext{
Received Feb. 20, 2015; revision accepted Mar. 23, 2015.

For correspondence or reprints contact: Geoffrey M. Currie, Charles Sturt

University, P.O. Box U102, CSU, Wagga Wagga, NS 2650, Australia.

E-mail: gcurrie@csu.edu.au

Published online May 8, 2015.

COPYRIGHT (c) 2015 by the Society of Nuclear Medicine and Molecular Imaging, Inc.
}

of practice as developed economies (such as the United States, the United Kingdom, Canada, Australia, and much of Europe).

A large volume of peer-reviewed articles is published across a multitude of different disciplines every year (I). These articles form the foundation of a profession's knowledge, and the bibliometric distribution reflects publication patterns of authors in that profession $(1,2)$. An author's productivity and research impact are often used for promotion and recruitment, both academically and clinically, or for grant decisions (2-9). Productivity is taken as a measure of the number of articles published by an author $(3,5,6,9-12)$ but should be limited to appropriately peer-reviewed journals. An article's impact or quality is more difficult to measure $(3,5,12)$. Various methods of citation analysis allow for the quantification of an author's or a publication's quality and impact (11). Nonetheless, they have limitations and inherent dangers if used incorrectly. As a result, the validity and reliability of such metrics are being increasingly questioned $(3,11,13)$, including citation numbers, the h-index, and the RG score on ResearchGate.

Although there have been studies on the publication productivity and patterns of radiographers and radiation therapists (RTs), to our knowledge there have been no studies on nuclear medicine technologists (NMTs) or medical radiation science (MRS) as a whole $(2,14)$. A recent study examining productivity in radiography and radiation therapy provided useful insights into publications; however, the study was flawed because of sampling biases, including no deliberate sampling of NMTs, incidental inclusion of NMT authors, and omission of any sampling from the United States (2,14). Furthermore, key MRS journals were omitted (e.g., JNMT), and no sampling was undertaken outside MRS journals.

\section{MATERIALS AND METHODS}

This study focused on author productivity and publication patterns in MRS to provide insight into the global status of MRS and, in particular, nuclear medicine technology. The aim was to evaluate the contribution of NMTs to the wider MRS knowledge base and to highlight advanced practice approaches and development opportunities. This study was an analysis of bibliometric data.

Bibliometric data were accumulated from the Journal of Medical Imaging and Radiation Sciences (JMIRS), The Radiographer (rebranded as the Journal of Medical Radiation Sciences [JMRS]), Radiography, The South African Radiographer, Radiologic Technology, Radiation Therapist, and Journal of Nuclear Medicine Technology. These journals were chosen because of use of the first 4 in the previous study, their global representation (Canada, Australia, 
the United Kingdom, South Africa, and the United States), and their representation of the MRS disciplines $(2,14)$. The data were collected over a 5-y period (2009-2013), and editorials, correspondence, and educational reviews were excluded. The medical radiation technologist authors with the highest productivity were further examined using PubMed and ResearchGate to produce a list of their articles published between 2009 and 2013. A cutoff of 3 per year was used to be more broadly inclusive than the 1-per-year cutoff used in the previous study.

The most productive authors were from the United States, United Kingdom, Canada, and Australia, where a similar relative workforce relationship exists (if not in absolute or per capita terms). A subanalysis was undertaken using data from the Australian Health Practitioner Regulation Agency (15). National registration in Australia is compulsory for employment in any capacity, and thus, the data of the Australian Health Practitioner Regulation Agency reliably capture $100 \%$ of the MRS workforce. A correction factor based on the relative workforce sizes was used to compare productivity per member of the registered workforce. Although reliable data could not be sourced for the United Kingdom, the United States, or Canada, these factors were thought to be similar across those countries. Using radiographers as the baseline (correction factor of $\times 1.0$ ), RTs were corrected with $\times 4.91$ and NMTs with $\times 11.01$ (calculated as the number of radiographers divided by the number of RTs or NMTs).

\section{RESULTS}

During the sampling period, 969 articles were published in the 7 identified key peer-reviewed journals, and these articles were written by 2,923 authors (2,083 unique authors). In total, 165 medical radiation technologists were identified who had published 3 or more articles between 2009 and 2013 (range of 3-58, with a total of 1,316 articles). Of these authors, $55.2 \%$ (91/165) were radiographers, $35.2 \%$ (58/165) were RTs, and $9.6 \%(16 / 165)$ were NMTs. Overall, the majority of the most prolific authors were academics $(104 / 165 ; 63.0 \%)$ (Table 1). Broken into specializations, the majority of the most prolific NMTs were academics (11/16; 68.8\%), with only $18.7 \%$ (3/16) and $12.5 \%$ (2/16) of these authors being from clinical affiliations or clinical with academic affiliations, respectively. Of the radiographers, $80.2 \%$ (73/91) had academic affiliations, $8.8 \%$ (8/91) had clinical affiliations, and $11.0 \%$ (10/91) had clinical with academic affiliations. In contrast, most RTs had clinical positions with academic affiliations $(22 / 58 ; 37.9 \%)$. Of the remaining RTs, $34.5 \%$ (20/58) had academic affiliations and 27.6\% (16/58) had clinical affiliations.

Overall, the majority of the most prolific authors were from the United Kingdom $(45 / 165 ; 27.3 \%)$ (Table 2), and this find- ing reflected the predominance of radiography publications, the U.K. dominance of the radiography publication metrics, and the relative size of that specialization in MRS. The majority of the most prolific radiographer authors were from the United Kingdom (38/91; 41.8\%), whereas the most prolific NMT authors were from the United States $(10 / 16 ; 62.5 \%)$ and the most prolific RT authors were from Canada (28/58; 48.3\%). The data corrected for workforce size (in parentheses in Table 2) highlight this specialization/country-of-origin relationship. The distribution of articles written by the most prolific authors among the 7 key MRS journals demonstrated a bias in NMTs toward JNMT, RTs toward JMIRS, and radiographers toward Radiography (Table 3), and this finding is consistent with the dominance of those professions in the country of origin of the journals. It should be noted that these figures reflect the authors' professional background rather than the article content, with many articles written that are specialization-neutral (e.g., education- or research-based, or indeed cross disciplinary).

There were 16 NMTs who featured among the most prolific 165 authors. As discussed previously, most of the NMT authors were from the United States (Table 2); however, the NMT authors originating from Australia contributed to more articles. The NMT authors originating from the United States published most of their articles in JNMT, the journal from the United States, whereas the Australian NMT authors published more widely. The absence of Canadian NMTs from the most prolific authors suggests publication inactivity.

As previously discussed, there were 58 RTs who featured among the 165 most prolific authors, and the majority was from Canada (Table 2). Within the 7 journals, the Canadian authors published articles primarily in JMIRS, the key international journal based in Canada. Australian RTs were also productive, with a large number of articles being published over a variety of journals. In comparison, RT authors from the United Kingdom and the United States were relatively inactive.

The majority of the most prolific radiographer authors were from the United Kingdom (Table 2). Radiographer authors from the United Kingdom published their articles primarily in Radiography (a U.K. journal). The radiographer authors from the United States also published primarily in their own journal, Radiologic Technology. This patriotism is consistent with the NMTs and RTs from the United States. As with the Australian NMT and RT authors, Australian radiographer authors published more widely,

TABLE 1

Number of Most Prolific Authors vs. Affiliation

\begin{tabular}{lcccc}
\hline \multirow{2}{*}{\multicolumn{1}{c}{ Affiliation }} & \multicolumn{4}{c}{ Number of most prolific authors } \\
\cline { 2 - 4 } & NMTs & RTs & Radiographers & Total \\
\hline Academic & $11(68.8 \%)$ & $20(34.5 \%)$ & $73(80.2 \%)$ & $104(63.0 \%)$ \\
Clinical with academic & $2(12.5 \%)$ & $22(37.9 \%)$ & $10(11.0 \%)$ & $34(20.6 \%)$ \\
Clinical & $3(18.7 \%)$ & $16(27.6 \%)$ & $8(8.8 \%)$ & $27(16.4 \%)$ \\
\hline
\end{tabular}


TABLE 2

Number of Most Prolific Authors vs. Country

\begin{tabular}{lcccc}
\hline & \multicolumn{4}{c}{ Number of most prolific authors } \\
\cline { 2 - 5 } \multicolumn{1}{c}{ Country } & NMTs & RTs & Radiographers & Total \\
\hline United Kingdom & $1(11)$ & $6(29)$ & 38 & 45 \\
\hline Australia & $4(44)$ & $17(83)$ & 17 & 38 \\
Canada & $0(0)$ & $28(137)$ & 2 & 30 \\
\hline United States & $10(110)$ & $4(20)$ & 15 & 29 \\
\hline Nigeria & 0 & 0 & 9 & 9 \\
\hline South Africa & 0 & 2 & 2 & 4 \\
\hline New Zealand & 0 & 1 & 2 & 3 \\
\hline Sweden & 0 & 0 & 2 & 2 \\
\hline Ireland & 1 & 0 & 1 & 2 \\
\hline Malaysia & 0 & 0 & 1 & 1 \\
\hline Uganda & 0 & 0 & 1 & 1 \\
\hline Malta & 0 & 0 & 1 & 1 \\
\hline
\end{tabular}

Relative workforce corrections are in parentheses using Australian data.

which could suggest a lack of international visibility of the Australian journal, the Journal of Medical Radiation Science. As with the Canadian NMTs, Canadian radiographer authors were relatively inactive.

After applying a correction factor (productivity per member of the registered workforce), we could compare the relative productivity of the 3 specializations of MRS. Overall, for the most prolific 165 authors the relative workforce corrections were 91 for radiographer authors $(91 \times 1), 285$ for RT authors $(58 \times 4.91)$, and 176 for NMT authors $(16 \times 11.01)$. This finding demonstrates that, overall, radiography has the fewest authors publishing, compared with the relative workflow sizes, likely because of issues relating to research culture and opportunity.

The most prolific 16 NMT authors were from 4 different countries (Table 4). Although most of the NMT authors were from the United States, the top 5 NMT authors were from Australia, Ireland, and the United Kingdom. The United States had a large number of authors publishing a handful of articles, whereas Australia, Ireland, and the United Kingdom had 1 or 2 highly productive authors. The majority of NMT authors were academics, suggesting that the academic institutions in these countries are highly productive whereas the nuclear medicine clinical institutions are less productive. The RT authors who featured among the most prolific 165 were from 6 different countries (Table 5); however, only authors from Australia or Canada featured among the most prolific 16 RT authors (Table 6). This finding suggests that Australian and Canadian RTs are highly productive whereas RTs from other countries are relatively inactive. The 14 most prolific radiographer authors were from 4 different countries-Australia, the United Kingdom, Ireland, and Nigeria (Table 7) — of the 12 countries in total (Table 2). As with the NMT authors, the most prolific 5 radiographer authors were from Australia, the United Kingdom, and Ireland, again suggesting that although Australia may not have the most authors, the Australian authors were very productive. Like the most prolific NMT authors, the most prolific radiographer authors were mostly academics.

\section{DISCUSSION}

The most prolific authors were for the most part academics $(83.6 \%)$, verifying that academic institutions are highly influential to research in MRS. The radiographer and NMT authors were consistent with this trend, with most having academic affiliations ( $81.3 \%$ and $91.2 \%$, respectively). The RT authors, however, were more evenly spread across the different affiliations. Although $72.4 \%$ had an academic affiliation, $65.5 \%$ also had a clinical affiliation, suggesting that RT research is more strongly clinically driven than radiographer and NMT research. This finding is likely to reflect advanced practice across both clinical and academic positions in the RT specialization and the tendency to appoint an RT research coordinator in some larger clinical departments. This observation also creates an opportunity to refine approaches and develop strategies to enhance research output among radiographers and NMTs. Indeed, academic institutions might be encouraged to formalize clinical affiliations to enhance publication productivity. The professions and journals would also benefit from encouraging and supporting this type of strategy.

TABLE 3

Distribution of Articles Written by Each Type of Author

\begin{tabular}{|c|c|c|c|}
\hline \multirow[b]{2}{*}{ Journal } & \multicolumn{3}{|c|}{ Number of articles } \\
\hline & NMT authors & RT authors & Radiographer authors \\
\hline JNMT & 45 & 0 & 3 \\
\hline JMRS & 9 & 54 & 42 \\
\hline Radiography & 8 & 35 & 197 \\
\hline$J M I R S$ & 7 & 108 & 31 \\
\hline The South African Radiographer & 0 & 4 & 26 \\
\hline Radiologic Technology & 0 & 5 & 54 \\
\hline Radiation Therapist & 0 & 8 & 3 \\
\hline ResearchGate & 28 & 108 & 198 \\
\hline PubMed & 48 & 165 & 130 \\
\hline Total & 145 & 487 & 684 \\
\hline Workforce correction & 1,596 & 2,391 & 684 \\
\hline
\end{tabular}


TABLE 4

The 16 Most Prolific NMT Authors

\begin{tabular}{|c|c|c|c|c|}
\hline Author & Number of articles & Overall rank & Country & Affiliation \\
\hline Currie & 31 & 5 & Australia & Academic \\
\hline Wheat & 23 & 9 & Australia & Academic \\
\hline Rainford & 21 & 12 & Ireland & Academic \\
\hline Knapp & 13 & 22 & United Kingdom & Academic \\
\hline Munn & 12 & 27 & Australia & Academic \\
\hline Folks & 11 & 30 & United States & Academic \\
\hline Bolus & 4 & 92 & United States & Academic \\
\hline Gilmore & 4 & 92 & United States & Academic \\
\hline Grantham & 4 & 92 & United States & Clinical with academic \\
\hline Mosman & 4 & 92 & United States & Clinical \\
\hline Farrell & 3 & 122 & United States & Clinical \\
\hline Hubble & 3 & 122 & United States & Academic \\
\hline James & 3 & 122 & Australia & Academic \\
\hline Jones & 3 & 122 & United States & Clinical \\
\hline Macci-Bires & 3 & 122 & United States & Academic \\
\hline Martin & 3 & 122 & United States & Clinical with academic \\
\hline
\end{tabular}

The most prolific authors were predominantly from the United Kingdom (27.3\%), as primarily reflects the high level of engagement of U.K. radiographers in publication. Indeed, the United Kingdom represented $41.8 \%$ of the most prolific radiographer authors, and radiographers represented $55.2 \%$ of the most prolific authors. However, a large volume of radiography publications presented generic material on topics such as education, patient care, or radiation safetytopics that are equally apt for RTs and NMTs. Consequently, many of these articles would be equally valid across all MRS journals, indicating an opportunity for journals such as JNMT to expand international scope, increase the number of publications, and widen the author pool. As reflected in Table 2, the United Kingdom did not have a strong performance among NMTs and RTs. Canada had $48.3 \%$ of the most prolific RT authors, but Canadian NMTs and radiographers were essentially inactive. The United States represented $62.5 \%$ of the most prolific NMT authors, but RTs were largely inactive and radiographers only moderately active. Australia tended to perform moderately across all specializations.

The publication patterns of the most prolific authors provide an interesting insight. Although there were only 16 NMT authors in the most prolific author list, collectively they produced 145 articles (9.1 articles per author for the 5-y window). As illustrated in Table 3, there was a bias in NMTs toward JNMT, although $52.4 \%$ of nuclear medicine technology articles were published outside the 7 key MRS journals. RTs comprised $35.2 \%$ of the most prolific authors, with an average of 8.4 articles per top author over the sampling period. RTs demonstrated a bias toward JMIRS, reflecting in part the predominance of Canada in the radiation therapy publication market. Similar to nuclear medicine technology articles, $56.1 \%$ of radiation therapy articles were published outside the $7 \mathrm{key}$ MRS journals. These figures for nuclear medicine technology and radiation therapy are likely to represent the advanced status of research in those specializations compared with radiography and the broader collaboration of transferrable research across discipline lines. For example, NMTs and RTs publishing in medical and basic science journals reflect content and collaboration beyond a technical readership alone. This also represents an important opportunity to refine strategy for key journals such as $J N M T$ and JMIRS. If $56 \%$ of NMT authors are publishing outside the key MRS journals, there is a large pool of highquality work that could be attracted back to JNMT or $J M I R S$. Indeed, the evolution of JNMT to a broader nuclear

TABLE 5

Distribution of Articles Written by RTs vs. Country

\begin{tabular}{|c|c|c|c|c|c|}
\hline \multirow[b]{2}{*}{ Journal } & \multicolumn{5}{|c|}{ Number of articles } \\
\hline & Australia & Canada & United Kingdom & United States & Other \\
\hline JMRS & 49 & 0 & 1 & 1 & 3 \\
\hline Radiography & 19 & 3 & 13 & 0 & 0 \\
\hline JMIRS & 13 & 95 & 0 & 0 & 0 \\
\hline The South African Radiographer & 0 & 0 & 0 & 0 & 4 \\
\hline Radiologic Technology & 1 & 0 & 0 & 4 & 0 \\
\hline Radiation Therapist & 0 & 2 & 0 & 6 & 0 \\
\hline ResearchGate & 55 & 38 & 10 & 1 & 4 \\
\hline PubMed & 37 & 118 & 1 & 8 & 1 \\
\hline Total & 174 & 256 & 25 & 20 & 12 \\
\hline
\end{tabular}


TABLE 6

The 16 Most Prolific RT Authors

\begin{tabular}{|c|c|c|c|c|}
\hline Author & Number of articles & Overall rank & Country & Affiliation \\
\hline Holden & 42 & 2 & Canada & Clinical with academic \\
\hline Halkett & 37 & 3 & Australia & Clinical with academic \\
\hline Cox & 26 & 7 & Australia & Academic \\
\hline Rosewall & 24 & 8 & Canada & Clinical \\
\hline Mitera & 21 & 12 & Canada & Clinical with academic \\
\hline Dempsey & 16 & 14 & Australia & Academic \\
\hline Jon & 15 & 15 & Canada & Clinical with academic \\
\hline Middleton & 15 & 15 & Australia & Clinical \\
\hline $\mathrm{Li}$ & 13 & 22 & Canada & Clinical with academic \\
\hline Wong & 13 & 22 & Canada & Clinical with academic \\
\hline Owen & 12 & 27 & Australia & Clinical \\
\hline Frantzis & 11 & 30 & Australia & Clinical \\
\hline Bolderston & 10 & 33 & Canada & Clinical with academic \\
\hline Di Prospero & 10 & 33 & Canada & Clinical with academic \\
\hline Findlay & 10 & 33 & Australia & Academic \\
\hline Higgins & 10 & 33 & Canada & Clinical with academic \\
\hline
\end{tabular}

medicine focus rather than being solely technology-based may have driven NMT authors to other publications.

In contrast to NMTs and RTs, radiographers demonstrated a trend toward Radiography, reflecting in part the local support of their journal in the United Kingdom and the lack of translatable reach to nondiscipline journals ( 7.5 articles per author over the sampling frame). Both radiation therapy and nuclear medicine technology are relatively small specializations compared with radiography. A disproportionately high number of the most prolific authors were among NMTs and RTs, compared with radiographers. Corrected for workforce size, RTs demonstrated the highest degree of productivity among the most prolific authors $(2,391)$, followed by NMTs $(1,596)$ and radiographers $(684)$. Again, these relationships reflect the advanced practice-driven research culture within the RT and NMT specializations, the evolving nature of technology in the same, and actual accessibility to research opportunities for technical staff.

A closer examination of the articles associated with the most prolific authors in specific specializations revealed several interesting insights. Although the majority of the most prolific NMT authors were in the United States, Australian NMTs published more articles during the study period. U.S. NMTs predominantly supported JNMT, whereas Australian NMTs published more broadly across the 7 MRS journals and outside those journals (Table 8). Ireland had 21 articles, but all were from the same author. Australia had a few very productive authors (17.3 articles per author), whereas the U.S. NMTs had a larger number of less productive authors (4.2 articles per author). Canada was the most productive for publications by RTs, who predominantly supported the JMIRS, whereas Australian RTs, like NMTs, published more broadly (Table 5). Although Australian RTs published widely across a variety of journals, within the 7 journals the majority of articles were published in JMRS. Australian RTs (10.2 articles per author) and Canadian RTs (9.1 articles per author) were the most productive, whereas RTs in the United States (5.0 articles per author) and United Kingdom (4.2 articles per author) were somewhat less productive. The most prolific radiographer authors were from a more diverse array of countries, although most originated in the United Kingdom (Table 9). The U.K. radiographers published mostly

TABLE 7

The 14 Most Prolific Radiographer Authors

\begin{tabular}{lccc}
\hline Author & Number of articles & Overall rank & Country \\
\hline Brennan & 58 & 1 & Australia \\
McEntee & 32 & 4 & Australia \\
Hogg & 31 & 5 & United Kingdom \\
McNulty & 23 & 9 & Academic \\
Reed & 22 & 11 & Academic \\
Smith & 15 & 15 & Australia \\
Snaith & 15 & 15 & Australia \\
Egbe & 14 & 19 & United Kingdom \\
French & 14 & 19 & Nigeria \\
Poulos & 14 & 19 & Canada \\
Jonker & 13 & 22 & Australia \\
Ugwu & 13 & 22 & United Kingdom \\
Thoirs & 12 & 27 & Clinical \\
England & 11 & 30 & Academic \\
\hline
\end{tabular}


TABLE 8

Distribution of Articles Written by NMTs vs. Country

\begin{tabular}{lrccc}
\hline & \multicolumn{4}{c}{ Number of articles } \\
\cline { 2 - 5 } \multicolumn{1}{c}{ Journal } & Australia & Ireland & $\begin{array}{c}\text { United } \\
\text { Kingdom }\end{array}$ & $\begin{array}{c}\text { United } \\
\text { States }\end{array}$ \\
\hline JNMT & 14 & 0 & 0 & 31 \\
\hline JMRS & 9 & 0 & 0 & 0 \\
Radiography & 2 & 4 & 2 & 0 \\
JMIRS & 5 & 2 & 0 & 0 \\
\hline ResearchGate & 12 & 8 & 5 & 3 \\
PubMed & 27 & 7 & 6 & 8 \\
\hline Total & 69 & 21 & 13 & 42 \\
\hline
\end{tabular}

in the United Kingdom-based Radiography, and the U.S. radiographers published the greater part of their articles in the United States-based Radiologic Technology. Many of these articles are generic and afford an opportunity for JNMT to attract authors from a new market. Like Australian RTs, Australian radiographers published more widely over a variety of journals but within the 7 key MRS journals. The top publishing radiographer from Ireland was the most productive, with 23 articles; however, as with NMTs, this was only a single author. Australian radiographers were highly productive (13.4 articles per author), suggesting a smaller number of authors who are highly productive compared with the United Kingdom (6.2 articles per author) and the United States (4.6 articles per author), which had a larger number of authors with a lower publication rate per author. Indeed, the relatively small population of Australia (and associated MRS workforce size) would demand that a small absolute number of authors be highly productive for Australia to command a footprint on the international stage.

Australia and the United States dominated the NMT author list; Canada and Australia, the RT author list; and Australia and the United Kingdom, the radiographer author list. More than $50 \%$ of the articles from the most prolific 165 authors originated outside the 7 key MRS journals, highlighting the importance of appropriate sampling but also the diverse publication patterns of medical radiation technologists. An author with an academic appointment is more likely to publish outside the 7 key MRS journals than are clinical authors. This trend likely reflects the impor- tance placed on impact factors by academic institutions in decisions on performance and promotion. Authors from Australia and Canada are also more likely to publish outside the 7 key MRS journals, as may reflect the level of professionalism, advanced practice, and cross-disciplinary research in these countries.

\section{Study Limitations}

The workforce corrections were based on the national registration data of the Australian Health Practitioner Regulation Agency, which reliably captures $100 \%$ of the Australian MRS workforce. Because reliable data could not be sourced for the United Kingdom, the United States, or Canada, the Australian data were used for extrapolation. Although the workforce sizes vary substantially among these countries, similarities in education, health care systems, cultural factors, and social factors were thought to lead to consistent relative relationships between the professions. It is probable, however, that there may be variations in the correction factors for each country. Unsubstantiated figures suggest that the radiography workforce in the United Kingdom is relatively larger than the numbers used in this study; if so, the corrections are likely to underestimate the real impact of the smaller specializations (NMT and RT) in the publication domain. Nonetheless, for the purposes of this study and associated metrics, the margin of error was considered negligible.

\section{Recommendations}

Specific recommendations depend on the perspective of the reader. From a clinical perspective, these results support a broader engagement in research across MRS. There is scope to enhance productivity by active engagement in clinical research; in Canada, particularly, the radiation therapy model is a sound one on which to build clinical productivity among NMTs, radiographers, and RTs outside Canada.

From an academic or higher-education perspective, increased visibility and productivity could be achieved by greater collaboration among colleagues and within the clinical domain. Furthermore, there is enormous potential within radiography for increased productivity, which may evolve as collaboration with nuclear medicine technology expands

TABLE 9

Distribution of Articles Written by Radiographers vs. Country

\begin{tabular}{|c|c|c|c|c|c|c|}
\hline \multirow[b]{2}{*}{ Journal } & \multicolumn{6}{|c|}{ Number of articles } \\
\hline & Australia & Canada & Ireland & United Kingdom & United States & Other \\
\hline JNMT & 0 & 0 & 0 & 3 & 0 & 0 \\
\hline JMRS & 34 & 1 & 0 & 4 & 0 & 3 \\
\hline Radiography & 39 & 1 & 2 & 131 & 1 & 23 \\
\hline JMIRS & 13 & 4 & 0 & 4 & 5 & 5 \\
\hline The South African Radiographer & 0 & 0 & 0 & 2 & 0 & 24 \\
\hline Radiologic Technology & 5 & 0 & 0 & 3 & 42 & 4 \\
\hline Radiation Therapist & 0 & 0 & 0 & 0 & 3 & 0 \\
\hline ResearchGate & 66 & 9 & 21 & 58 & 4 & 40 \\
\hline PubMed & 71 & 3 & 0 & 29 & 14 & 13 \\
\hline Total & 228 & 18 & 23 & 234 & 69 & 112 \\
\hline
\end{tabular}


through developments in hybrid technology. Indeed, the research culture within nuclear medicine technology may positively drive the research culture within radiography. The nuclear medicine technology and radiation therapy model applied to radiography would substantially increase overall MRS visibility and productivity. Thus, opportunities for engagement in research within radiography need to be explored and the underlying research culture enhanced. Furthermore, formal collaborations between academics and clinical departments represent a key strategy in enhancing productivity, particularly in nuclear medicine technology and radiography.

From an international perspective, larger economies such as the United States and the United Kingdom should adopt the Australian model to engage more productively and across all specializations. In particular, specific strategies need to be developed in Canada for nuclear medicine technology and radiography research, in the United Kingdom for nuclear medicine technology and radiation therapy research, and in the United States for increasing relative productivity across all specializations. Many of these opportunities can be exploited by developing advanced practice models and instilling a research culture within industry, perhaps starting during undergraduate study. This possibility is reflected, in part, in a recent white paper published in JNMT (16).

From a journal perspective, there are several opportunities to develop strategic plans to capture a larger market share. The key MRS-based journals need to recognize that more than $50 \%$ of publications are outside their scope. Consequently, there is an immediate opportunity to increase output by increasing the profile and visibility of the journal. That is, if any given MRS journal is PubMed-listed with a Journal Citation Reports impact factor of significant value, MRS authors may be less inclined to publish outside MRS. There is also scope for journals to support less productive areas to increase publication rates. In the United States, publication rates are relatively low, and journals such as JNMT typically survive on authors from outside the United States or non-MRS authors. In particular, JNMT needs to reclaim its status as the key outlet for technologists who wish to publish. Organic growth in publication rates across all MRS specializations in the United States is strongly recommended.

From a discipline perspective, MRS is highly visible and productive. Nonetheless, with more than 50\% of MRS publications being outside the MRS-specific journals, the overall impact and footprint of MRS is likely to be underrated. Individual professionals do not always have the time or capacity to read broadly enough to source and read all related articles. The real impact of MRS research may be lost if the broader target audience is not directly engaged. Nonetheless, publication outside MRS journals may reflect transdisciplinary research that targets a broader audience. Although the strategies above suggest that journals should draw those publications back into MRS journals, several barriers prohibit immediate or widespread success (e.g., transdisciplinary collaboration and impact factors). Therefore, to ensure that members of the profession are exposed to and appreciate the strength of MRS productivity, it is recommended that each edition of at least one major MRS journal include a section that summarizes publications in non-MRS journals. This goal could be achieved by tracking the publications of key authors identified in this article.

\section{CONCLUSION}

Through active research, medical radiation technologists contribute significantly to the knowledge base of both the MRS professions and the wider health care community. NMTs demonstrate a high degree of productivity both absolutely and relatively, particularly in Australia and the United States. $J N M T$ is the key nuclear medicine journal with international visibility, although more than half of nuclear medicine technology publications occur in non-medical radiation technology journals. NMTs have a productive research culture and command a large footprint within and outside the key MRS journals, contributing significantly to the research and knowledge quanta of the health and medical sciences.

\section{DISCLOSURE}

No potential conflict of interest relevant to this article was reported.

\section{REFERENCES}

1. Talukdar D. Patterns of research productivity in the business ethics literature: insights from analyses of bibliometric distributions. J Bus Ethics. 2011;98:137-151.

2. Snaith BA. An evaluation of author productivity in international radiography journals 2004-2011. J Med Imaging Radiat Sci. 2013;60:93-99.

3. Adams AB, Simonson D. Publications, citations, and impact factors of leading investigators in critical care medicine. Respir Care. 2004;49:276-281.

4. Currie GM. Impact factors in medical radiation science journals. J Med Imaging Radiat Sci. 2014;45:70-71.

5. Murphy CG, O'Sullivan ME. A five-year review of publication productivity among Irish orthopaedic units. Ir J Med Sci. 2011;180:691-695.

6. Pintér A. Changing trends in authorship patterns in the JPS: publish or perish. $J$ Pediatr Surg. 2013;48:412-417.

7. Post RE, Weese TJ, Manious AG III, Weiss BD. Publication productivity by family medicine faculty: 1999 to 2009. Fam Med. 2012;44:312-317.

8. Richter RR, Schlomer SL, Krieger MM, Siler WL. Journal publication productivity in academic physical therapy programs in the United States and Puerto Rico from 1998 to 2002. Phys Ther. 2008;88:376-386.

9. Rigg LS, McCarragher S, Krmenec A. Authorship, collaboration, and gender: fifteen years of publication productivity in selected geography journals. Prof Geogr. 2012;64:491-502.

10. Englesbe MJ, Lynch RJ, Sung RS, Segev DL. Time-trends in publication productivity of young transplant surgeons in the United States. Am J Transplant. 2012;12:772-778.

11. Nightingale JM, Marshall G. Citation analysis as a measure of article quality, journal influence and individual researcher performance. Radiography. 2012;18:60-67.

12. Wootton R. A simple, generalizable method for measuring individual research productivity and its use in the long-term analysis of departmental performance including between-country comparisons. Health Res Policy Syst. 2013;11:2-14.

13. Currie GM, Wheat JM. Impact factors in nuclear medicine journals. J Nucl Med. 2007;48:1397-1400.

14. Snaith BA. Peer-review publication patterns: a comparison of international radiography journals. J Med Imaging Radiat Sci. 2013;44:37-43.

15. Statistics. Australian Health Practitioner Regulation Agency website. http://www. medicalradiationpracticeboard.gov.au/About/Statistics.aspx. Accessed April 14, 2015.

16. Holbrook S, Baldwin J, Gilmore CD, et al. Clinical research: the future of the molecular imaging technologist - a white paper presented by members of the graduate stakeholders committee of the SNMMI Technologist Section. J Nucl Med Technol. 2014;42:228-232. 